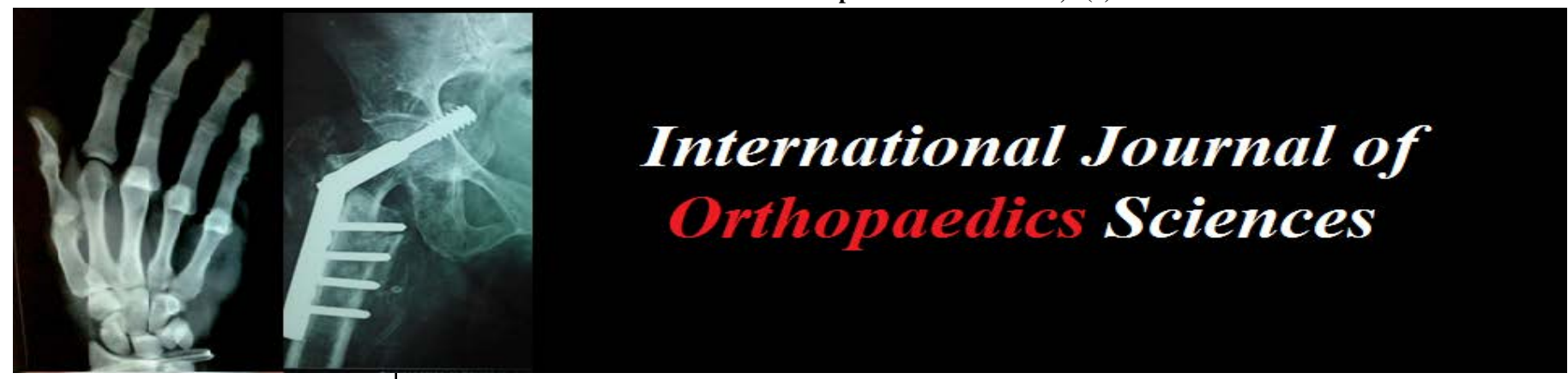

E-ISSN: 2395-1958

P-ISSN: 2706-6630

IJOS 2020; 6(4): 607-611

(C) 2020 IJOS

www.orthopaper.com

Received: 27-07-2020

Accepted: 23-08-2020

Dr. Madan Mohan Sahoo MS Orthopaedics, Associate Professor, Department of Orthopaedics, SCB Medical College and Hospital, Cuttack, Odisha, India

Dr. Udit Sourav Sahoo MS Orthopaedics, Senior Resident, Department of Orthopaedics, SCB Medical College and Hospital, Cuttack, Odisha, India

Dr. Satish Kumar Nanda MS Orthopaedics, Senior Resident, Department of Orthopaedics, SCB Medica College and Hospital, Cuttack, Odisha, India
Corresponding Author: Dr. Satish Kumar Nanda MS Orthopaedics, Senior Resident, Department of Orthopaedics, SCB Medical College and Hospital, Cuttack, Odisha, India

\section{Functional and radiological outcome of transforaminal lumbar inter body fusion in adult degenerative spondylolisthesis, a prospective cohort study}

\author{
Dr. Madan Mohan Sahoo, Dr. Udit Sourav Sahoo and Dr. Satish Kumar \\ Nanda
}

DOI: https://doi.org/10.22271/ortho.2020.v6.i4i.2393

\begin{abstract}
Background: Lumbar interbody fusion is an established and widely accepted procedure for degenerative spondylolisthesis. There are various fusion techniques and choosing one technique over the other remains controversial. Objective of our study was to review the long term functional and radiological outcome of traditional open transforaminal lumbar inter body fusion (TLIF) for this condition.

Design: Prospective cohort study.

Methods: This prospective study was conducted on 43 symptomatic adult degenerative spondylolisthesis patients, treated with traditional open TLIF and posterior instrumentation at a tertiary care centre with a mean age of 48.2years(range 26-59 year). Functional outcome and complications were assessed clinicoradiologically with a mean follow-up of 47.6 months (range18-65 months).

Results: 34 patients (80\%) were rated excellent, 7 patients (15\%) were rated good and 2 patients (5\%) were rated poor based on the pre and postoperative questionnaire scores. Visual analogue scale (VAS) pain score improved from 7.7 preoperatively to 2.5 at the latest follow-up. Preoperative Oswestry disability index (ODI) averaged 33.8 and postoperatively improved to 63.7 out of 100 . Radiographic bony fusion occurred in 41 patients (95\%) at final follow-up. Retropulsion of the cage into spinal canal occurred in one patient and no other major complications happened.

Conclusion: Traditional open TLIF achieved excellent functional outcome and high fusion rate in our study at a long-term follow-up, which is comparable to any other fusion technique.
\end{abstract}

Keywords: Transforaminal lumbar inter body fusion (TLIF), Degenerative spondylolisthesis, posterior lumbar inter body fusion (PLIF)

\section{Introduction}

Spondylolisthesis is the sagittal translational displacement of one vertebra with respect to other, and traditionally referred to the anterior displacement of the superior vertebra over the vertebra inferior to it. Degenerative spondylolisthesis is the most common variety in adults, characterised by age related disc degeneration, loss of disc height, sagittal translation which is often coupled with rotational deformity, facet joint degeneration and ligamentous laxity. Majority of the patients are asymptomatic. Lumbar decompression and inter-body fusion with instrumentation is commonly performed in symptomatic patients with radiculopathy and neurological claudications due to spinal stenosis in a small subgroup of these patients. Nearly $80 \%$ of the compressive, torsion, and shear forces are transmitted through the anterior column, hence successful bony fusion after disc removal is critical for a better functional outcome ${ }^{[1,2]}$. Reconstruction of the anterior column can be performed via anterior lumbar inter body fusion (ALIF), posterior lumbar inter body fusion (PLIF) and transforaminal lumbar inter body fusion (TLIF) techniques, or a combination of anterior and posterior approach.

Posterior approaches have gained more popularity due to lesser morbidity and a relatively easier learning curve and has evolved over the time with a number of options like isolated laminectomy, laminectomy with postero-lateral lumbar fusion (PLF), PLIF, TLIF with instrumentation producing a circumferential $360^{\circ}$ fusion. The original technique of PLIF has been modified by some surgeons over the time. TLIF was initially described by Harms and Rollinger ${ }^{[3]}$ in 1982, and gained wide acceptance after Harms and Jeszenszky in $1998^{[4]}$. 
It involves a transforaminal approach to the anterior interbody-space, theorising that the neurological complications with PLIF, specifically the dural tear, epidural scarring etc could be potentially avoided ${ }^{[5]}$. Currently TLIF has gained more popularity over traditional PLIF. However, considering the increased material expenses, longer operative time, and being a technically more challenging procedure than PLIF, evidence in favour of TLIF needs to be definitive, which rather has been inconclusive in a few recent studies ${ }^{[6}$, 7]. Recently surgeons have started minimally invasive TLIF, however it has a steeper learning curve, needs better surgical set-up and also not possible in advanced grades of listhesis.

We have done a prospective study on TLIF in all grades of spondylolisthesis with an aim to review the functional and radiological outcomes, identify procedure-specific complications, and determine any preoperative factors that may predict the outcome.

\section{Material and methods}

A prospective study was undertaken in our institute from July 2014 to February 2020 in which 43 symptomatic adult degenerative spondylolisthesis patients were treated with TLIF and posterior instrumentation. Patients with greater than Meyerding grade III, history of any previous surgery at lumbosacral level, kypho-scoliotic deformity, pathologic conditions of the lumbar spine and in age group of below 18 or above 70 were excluded from the study. Institutional ethical committee clearance was obtained beforehand.

Pre-operatively anteroposterior and lateral standing radiograph with flexion-extension dynamic lateral view and magnetic resonance imaging (MRI) of spine were done in all cases to evaluate the grade of slip, disc height, segmental instability, sagittal profile disc degeneration, spinal canal stenosis and neural compression. Postoperatively radiographs were obtained to assess the percentage of residual slip and percentage of reduction, height of disc space and intervertebral foramen, cage position, and bony fusion as per the criteria of Gertzbein [8], where continuity of trabecular pattern was the criteria for bony fusion. Visual analogue score (VAS) for pain and Oswestry disability index (ODI) of each patient were recorded preoperatively and at postoperative 3rd and 6th month, and 6 monthly thereafter.

\section{Operative procedure}

Patients were positioned prone with bolsters below chest and pelvis to keep lumbosacral spine in maximum extension, which helps in partial reduction of listhesis. With midline posterior incision and retraction of paraspinal muscles, pedicle screws were inserted. Either complete or hemilaminectomy with unilateral facetectomy of inferior facet of superior vertebrae was done according to the site and amount of neural compression. Reduction screws were used whenever reduction could not be achieved with positioning alone. Then annulus fibrosus was excised at foramen level in a rectangular fashion to remove the disc material and access disc space. After adequate curettage of end plates, a banana shaped cage of proper size was packed with morcelised autologous bone from resected elements and was introduced into the disc space and its position was confirmed on fluoroscopy.

Patients were ambulated on 2nd postoperative day. Brace support was given for 6-8 weeks after surgery. For first 6 weeks, patients were allowed limited walking and sitting for longer period or forward bending were restricted. At 6th week, progressive range of motion and back muscle strengthening exercises were started. By 3rd month, patients were allowed low impact activities as tolerated and normal activities were resumed at 6th month.

\section{Results}

Forty three patients were evaluated with a mean follow-up of 47.6 months (range18-65 months). Mean age at surgery was 48.2years (range 26-59 year) with 28 women (65\%) and 15men (35\%). Level of slip was seen more at L4-L5 level with 24 patients (55\% of total cases). Grade I slip was found in 26 cases (60\%), 15(35\%) had grade II and only 2 cases (5\%) had grade III slip (Fig. 1a, 1b).

34 patients (80\%) were rated excellent, 7 patients $(15 \%)$ were rated good and 2 patients (5\%) were rated poor based on the pre and postoperative questionnaire scores, which included combined pain and activities of daily living (ADL) scores. Pain level on a 10 point visual analogue scale (VAS) improved from a preoperative mean value of 7.7 to 2.5 at the latest follow up. No patients reported any postoperative pain greater than the preoperative level. Pre-operatively 39(90\%) patients were taking at least one nonsteroidal antiinflammatory drugs (NSAID) daily for pain and postoperatively 2(4.6\%) patients were taking NSAID at latest follow up. The preoperative Oswestry disability index (ODI) averaged 33.8 out of 100 and postoperatively increased to 63.7 out of 100 . No patients in our series became less able to perform activities of daily living (ADL) postoperatively. When asked if they would have the surgery again, based on their outcome, $35(80 \%)$ patients said they would. $70 \%$ of the total patients were having sciatica and radiculopathy symptoms which improved post-operatively in $90 \%$ of those. Radiographic bony fusion was evident in 41 patients(95\%) at final follow-up with obliteration of disc space anterior to the cages and continuous trabecular pattern across the vertebral bodies(Fig. 2). No demonstrable movement on flexion extension radiographs was found in any cases. One patient had radiographic evidence of a possible pseudoarthrosis but was asymptomatic in all the follow-ups.

Average operative time was 168.8 minutes and average blood loss was $288 \mathrm{ml}$.

\section{Complications}

In one patient, we found retropulsion and subsequent displacement of the cage into spinal canal, however the patient was followed up regularly and remained asymptomatic throughout (Fig. 3).

Four (9\%) cases developed infection but responded to judicious antibiotic therapy and one patient developed transient foot drop in immediate post-operative period, which recovered in course of time

Preoperatively, there were 28(65\%) patients working and 15(35\%) were not working because of backpain. Postoperatively, all the patients who were working returned to work and $13(85 \%)$ of 15 , who were not working because of backpain returned to light work postoperatively.

\section{Discussion}

Lumbar interbody fusion is a commonly performed surgical procedure for a variety of spinal conditions like spondylolisthesis, degenerative disc disease, trauma, and infections etc. ${ }^{[9]}$ Bone graft, cage, or cage with bone graft is packed into the intervertebral space after discectomy and curettage of endplates in this procedure, which leads to bony fusion. Goal of surgical treatment in degenerative spondylolisthesis are stabilization of the motion segment, neural decompression, restoration of disc height and sagittal plane translational and rotational alignment. Interbody fusion 
stabilizes the motion segment, gives a biomechanically stable anterior column, produces indirect foraminal decompression and restores the load-bearing capacity and disc height. ALIF, PLIF, TLIF are described techniques of interbody fusion and all have been reported to provide acceptable fusion rates and clinical outcomes in spondylolisthesis ${ }^{[10,11]}$. However still there remains a need to determine the optimal fusion technique, since each is having certain advantages and specific drawbacks.

Disadvantages of ALIF includes increased morbidity to the patient, injury to peritoneal and retroperitoneal organs or major blood vessels, sympathetic nerve injury, retrograde ejaculation and often needs a teamwork with vascular and general surgeons. Moreover, anterior fixation is mechanically inferior when compared to posterior fixation with pedicle screws. In PLIF procedure surgeon needs to retract the dural sheath medially at least to midline to approach the disc space. This is responsible for a greater incidence of nerve damage, dural tear, epidural scarring, post-operative neurogenic pain etc. [5] Again, PLIF is often limited between L3-S1 level, because retraction of nerves at a higher-level poses danger to conus medullaris and cauda equina. Humphreys et al. [5] found a higher incidence of neurological complications and radiculitis with PLIF and blamed greater medial retraction of the thecal sac for the same.

TLIF is a modification of the PLIF technique which can achieve a $360^{\circ}$ of circumferential fusion through a single posterolateral approach with minimal retraction of nerves, and thus reduces injury to central neural structures unlike that with PLIF, yet producing similar stability. This has been shown to reduce the incidence of postoperative radiculitis, eliminate epidural scarring and reduce intraoperative bleeding [12]. Average operative time was 168.8 minutes and average blood loss was $288 \mathrm{ml}$ in our study, supporting above mentioned evidences.

Challier et al. In their randomised controlled trial found that TLIF is associated with greater odds of bony fusion at two years follow-up ${ }^{[13]}$. We found radiographic bony fusion in 41 patients (95\%) at mean follow-up of 47.6 months (range18-65 months). No demonstrable movement on flexion extension radiographs was found in any cases. This higher rate is also supported in other studies ${ }^{[6,14]}$.

TLIF facilitates better decompression in foramen and lateral recess area, hence more suitable when radicular pain is the dominant symptom [6, 14, 15]. Our study also supports this finding with $30(70 \%)$ of the total patients were having sciatica and radiculopathy symptoms which improved postoperatively in 27(90\%) of those. Another advantage with TLIF is that it allows access to whole of the disc space through only unilateral approach even at cauda equina level. This is not possible with PLIF, because nerves can not be retracted safely at this level and discectomy and graft insertion is done in a bilateral fashion ${ }^{[5]}$. TLIF can also be augmented with posterolateral fusion and instrumentation through a single posterior approach like PLIF.

However the major disadvantage of TLIF is significant muscle retraction and dissection on lateral and upto far lateral level. A few studies have shown a significant association between longer operative time and perioperative complications, raising concern over TLIF ${ }^{[6,7,16-18]}$.

Minimally invasive transforaminal lumbar interbody fusion (MITLIF) has come up as a technical advancement to the established TLIF technique. Major advantage over open TLIF is no paraspinal muscle stripping, minimal soft tissue trauma, negligible blood loss, smaller surgical wounds, quicker rehabilitation and reduced postoperative pain ${ }^{[19,20]}$. However, it is difficult for spine surgeons to adapt to MITLIF for various reasons. It is technically challenging and has a steep learning curve. Instruments are costly and better set-up is needed. It takes more operative time than open TLIF. Contralateral side decompression is not possible with an unilateral MITLIF approach, which is a major disadvantage in patients with bilateral symptoms. Spondylolisthesis of more than Meyerding grade II requires bilateral MIS-TLIF and bilateral interbody cages, which increases the duration of surgery and morbidities associated with it. It also causes more radiation exposure than open TLIF or any other conventional lumbar fusion technique ${ }^{[19-21]}$.

Regarding clinical outcomes, measured using the VAS and ODI scores, most of the studies showed significant improvement in VAS (for back and leg pain) and ODI scores for both MITLIF and OTLIF, with no significant difference between techniques ${ }^{[16,20,22-29]}$. Immediate post-operative pain was less in MITILF patients ${ }^{[20,22]}$. However, studies reported no significant differences in the long-term functional outcomes, VAS pain scores, or ODI scores between the two techniques [20, 24-29]. Adogwa et al showed no significant difference in patient reported outcomes for back pain, leg pain, and functional status 1 and 2 years post-operatively in obese patients undergoing MITLIF or TLIF [29]. Similarly Terman et al showed significant improvement in pain and function in obese patients undergoing MITLIF and TLIF, with comparable results to non-obese patients with no significant differences between MITLIF and TLIF ${ }^{\text {[27] }}$. In our study, VAS pain score improved from a preoperative mean value of 7.7 to 2.5 at the latest follow up with a mean of 47.6 months. Similarly, the preoperative Oswestry disability index (ODI) averaged 33.8 out of 100 and postoperatively improved to 63.7 out of 100 at final follow-up. No patients in our series became less able to perform activities of daily living (ADL) postoperatively on long term follow-up basis. These findings of our study along with a long duration follow-up consolidates the evidence that conventional open TLIF can provide excellent pain relief and functional outcome comparable to MITLIF on a long term basis.

The complication rate comparing MITLIF and open TLIF shows heterogeneity among various studies. Many studies showed no significant difference between the techniques ${ }^{[25,28-}$ 29]. A Few studies have reported significantly higher complication rates with TLIF $[20,26,27]$. However, some studies like Lau et al showed that the complication rate tended to be higher for MITLIF than open TLIF ${ }^{\text {[23] }}$. Similarly Dhall et al. retrospectively examined 42 patients and reported a higher rate of complication with MITLIF, including screw misplacement and cage migration ${ }^{[28]}$. Both attributed the higher complications to steep learning curve and higher technical demands. However, a number of studies have found lower rate of systemic respiratory and urinary infections, general cardiopulmonary complications, durotomy, and wound infection in obese patients(BMI $>30 \mathrm{~kg} / \mathrm{m}^{2}$ ) undergoing MIS-TLIF, which was attributed to earlier patient mobilization and ambulation in them ${ }^{[21,23,26]}$.

Majority of studies showed no statistically significant difference in fusion rates between MITLIF and TLIF [16, 20, 22, 24, 25]. Villavicencio et al's retrospective study on 139 patients reported successful fusion with no difference between MITLIF and TLIF [24]. Seng et al. in retrospective comparative study found that patients undergoing TLIF showed slightly better fusion at 6 months and 2 years compared with patients undergoing MITLIF but the difference was not statistically significant, and fusion rates were similar at the 5-year follow-up ${ }^{[25]}$. Our study also has high fusion rate of $95 \%$ at mean follow-up of 47.6 months (range18-65 months).

Our study has limitations; a randomised controlled study comparing TLIF with PLIF or MITLIF could have given 
more strength to our conclusion.

\section{Conclusion}

In the treatment of adult degenerative spondylolisthesis, traditional open TLIF using single interbody cage and posterior instrumentation with pedicle screws along with contralateral decompression produced good to excellent clinical outcome in terms of pain control and higher fusion rate in long-term follow-up with minimal morbidity in our study. None of the patient had any major post-operative complications. We would recommend traditional open TLIF for degenerative spondylolisthesis and conclude that it's safety and efficacy is at par with any other fusion technique.

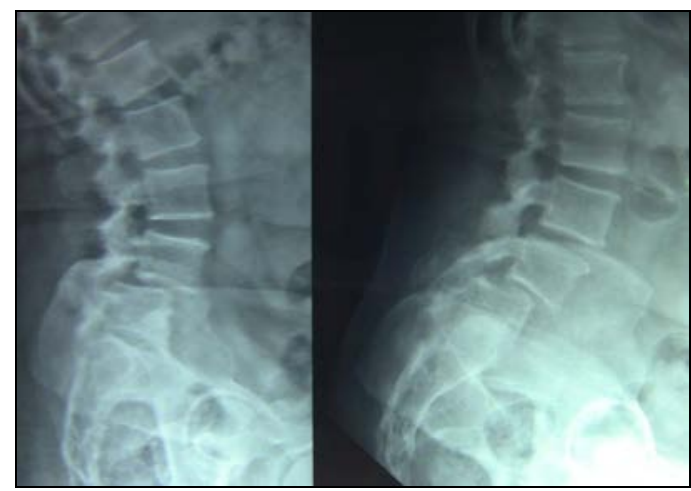

Fig 1a: Pre-operative X-ray radiograph, showing grade III slip at L45 level.

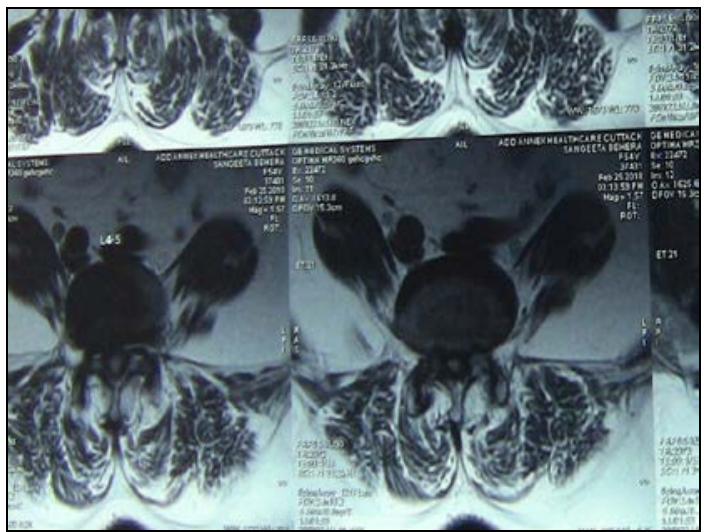

Fig 1b: Pre-operative MRI axial section, showing canal stenosis and far lateral compression on nerve root.

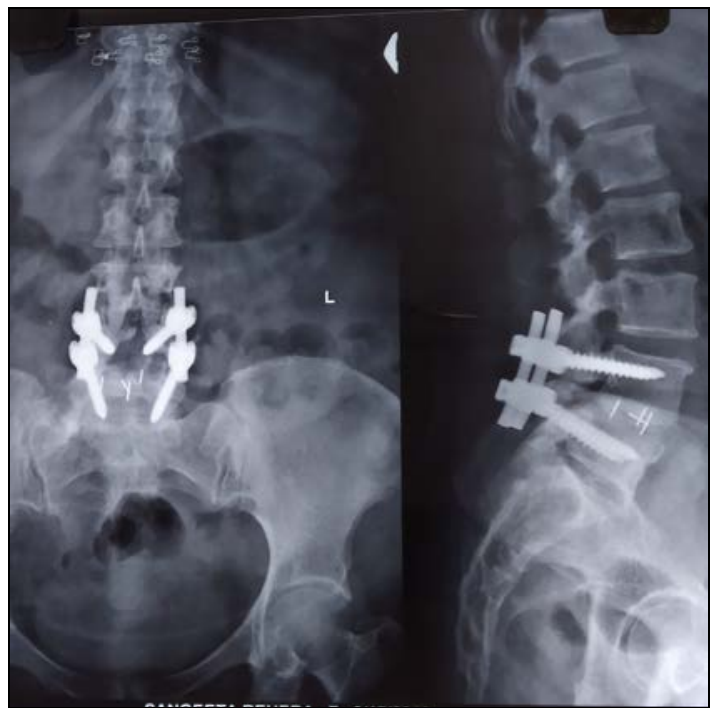

Fig 2: Post-operative X-ray radiograph, showing L4-5 level bony inter body fusion with a peek banana cage in the same patient.

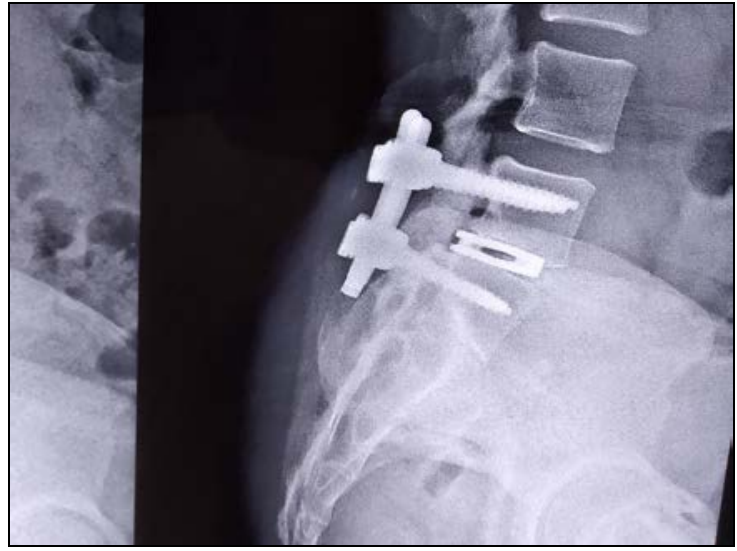

Fig 3a: Follow-up X-ray radiograph in a patient, showing retropulsion of the cage into spinal canal.
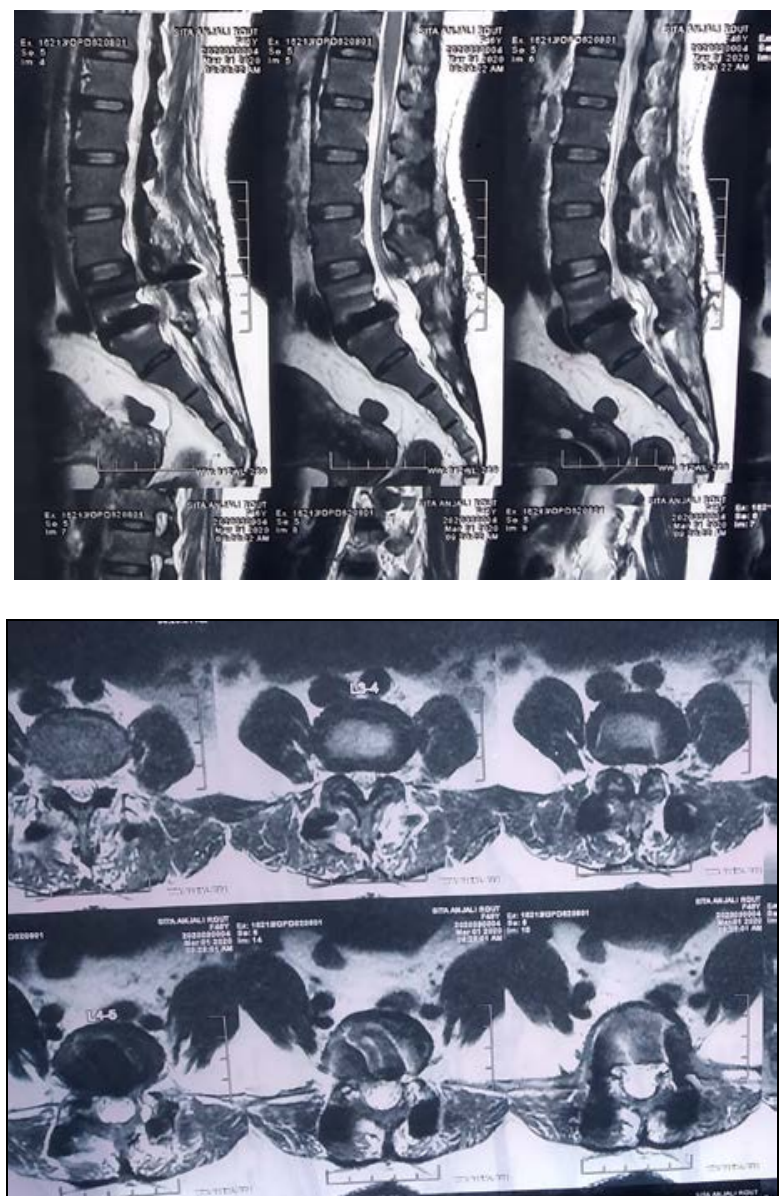

Fig 3b and Fig 3c: Follow-up MRI sagittal and axial sections of the same patient, showing retropulsion of the cage.

\section{Références}

1. Harms J. Screw-threaded rod system in spinal fusion surgery. Spine 1992;6:541-575.

2. Ishihara $\mathrm{H}$, Osada $\mathrm{R}$, Kanamori $\mathrm{M}$ et al. Minimum 10year followup study of anterior lumbar interbody fusion for isthmic spondylolisthesis. J Spinal Disord 2001;14:91-99.

3. Harms J, Rolinger H. A one-stager procedure in operative treatment of spondylolistheses: dorsal traction reposition and anterior fusion (author's transl). Z Orthop Ihre Grenzgeb 1982;120:343-7.

4. Harms JG, Jeszenszky D. The unilateral, transforaminal approach for posterior lumbar interbody fusion. Orthop Traumatol 1998;6:88-99.

5. Humphreys SC, Hodges SD, Patwardhan AG, et al. 
Comparison of posterior and transforaminal approaches to lumbar interbody fusion. Spine (Phila $\mathrm{Pa}$ 1976) 2001;26:567-71.

6. Abdu WA, Lurie JD, Spratt KF, et al. Degenerative spondylolisthesis: does fusion method influence outcome? Four-year results of the spine patient outcomes research trial. Spine (Phila Pa 1976) 2009;34:2351-60.

7. Gottschalk MB, Premkumar A, Sweeney K,. Posterolateral lumbar arthrodesis with and without interbody arthrodesis for L4- L5 degenerative spondylolisthesis. Spine (Phila Pa 1976) 2015;40:917-25.

8. Gertzbein SD, Betz R, Clements D, Errico $T$, Hammerberg $\mathrm{K}$, Robbins $\mathrm{S}$, et al. Semirigid instrumentation in the management of lumbar spinal conditions combined with circumferential fusion. A multicenter study. Spine (Phila Pa 1976). 1996;21(16):1918-25; discussion 1925-6.

9. Resnick DK, Choudhri TF, Dailey AT, et al. Guidelines for the performance of fusion procedures for degenerative disease of the lumbar spine. Part 7: intractable low-back pain without stenosis or spondylolisthesis. J Neurosurg Spine 2005;2:670-2.

10. Videbaek TS, Christensen FB, Soegaard R, et al. Circumferential fusion improves outcome in comparison with instrumented posterolateral fusion: long-term results of a randomized clinical trial. Spine (Phila $\mathrm{Pa}$ 1976) 2006;31:2875-80.

11. Christensen FB, Hansen ES, Eiskjaer SP, et al. Circumferential lumbar spinal fusion with Brantigan cage versus posterolateral fusion with titanium CotrelDubousset instrumentation: a prospective, randomized clinical study of 146 patients. Spine (Phila $\mathrm{Pa}$ 1976) 2002;27:2674-83.

12. Talia AJ, Wong ML, Lau HC, et al. Comparison of the different surgical approaches for lumbar interbody fusion. J Clin Neurosci 2015;22:243-51.

13. Challier V, Boissiere L, Obeid I, Vital JM, Castelain JE, Bénard A, et al. One-Level Lumbar Degenerative Spondylolisthesis and Posterior Approach: Is Transforaminal Lateral Interbody Fusion Mandatory?: A Randomized Controlled Trial With 2-Year Follow-Up. Spine (Phila Pa 1976) 2017;42(8):531-539.

14. Fujimori T, Le H, Schairer W, et al. Does transforaminal lumbar interbody fusion have advantages over posterolateral lumbar fusion for degenerative spondylolisthesis? Glob Spine J 2015; 5:102-9.

15. Hackenberg L, et al. Transforaminal lumbar interbody fusion: a safe technique with satisfactory three to five year results. Eur Spine J 2005;14:551-8.

16. Lee YS, Kim YB, Park SW, et al. Comparison of transforaminal lumbar interbody fusion with direct lumbar interbody fusion. Clin Radiol Results 2014;56:469-74.

17. Foley KT, Lefkowitz MA. Advances in minimally invasive spine surgery. Clin Neurosurg. 2002;49:449517.

18. Schwender JD, Holly LT, Rouben DP, et al. Minimally invasive transforaminal lumbar interbody fusion (TLIF): technical feasibility and initial results. J Spinal Disord Tech 2005;18:S1-6.

19. Park P, Foley KT, Cowan JA, Marca FL. Minimally invasive pedicle screw fixation utilizing O-arm fluoroscopy with computer-assisted navigation: Feasibility, technique, and preliminary results, Surgical Neurology International 2010;1:44.
20. Peng CW, Yue WM, Poh SY, et al. Clinical and radiological outcomes of minimally invasive versus open transforaminal lumbar interbody fusion. Spine (Phila $\mathrm{Pa}$ 1976). 2009;34:1385-9.

21. Xiao Y, Li F, Chen Q. Transforaminal lumbar interbody fusion with one cage and excised local bone. Arch Orthop Trauma Surg 2010;130:591-597.

22. Wang J, Zhou Y, Feng Zhang Z, et al. Comparison of the clinical outcome in overweight or obese patients after minimally invasive versus open transforaminal lumbar interbody fusion. J Spinal Disord Tech 2014;27:202-206.

23. Lau D, Khan A, Terman SW, et al. Comparison of perioperative outcomes following open versus minimally invasive transforaminal lumbar interbody fusion in obese patients. Neurosurg Focus 2013;35:E10.

24. Villavicencio AT, Burneikiene S, Roeca CM, et al. Minimally invasive versus open transforaminal lumbar interbody fusion. Surg Neurol Int 2010;1:12.

25. Seng C, Siddiqui MA, Wong KP, et al. Five-year outcomes of minimally invasive versus open transforaminal lumbar interbody fusion: a matched pair comparison study. Spine (Phila Pa 1976) 2013;38:204955.

26. Wong AP, Smith ZA, Stadler JA $3^{\text {rd }}$ et al. Minimally invasive transforaminal lumbar interbody fusion (MITLIF): surgical technique, long-term 4-year prospective outcomes, and complications compared with an open TLIF cohort. Neurosurg Clin N Am 2014;25:279-304.

27. Terman SW, Yee TJ, Lau D, et al. Minimally invasive versus open transforaminal lumbar interbody fusion: comparison of clinical outcomes among obese patients. J Neurosurg Spine 2014;20:644-52.

28. Dhall SS, Wang MY, Mummaneni PV. Clinical and radiographic comparison of mini-open transforaminal lumbar interbody fusion with open transforaminal lumbar interbody fusion in 42 patients with long-term follow-up. J Neurosurg Spine 2008;9:560-5

29. Adogwa O, Carr K, Thompson P, et al. A prospective, multi- institutional comparative effectiveness study of lumbar spine surgery in morbidly obese patients: does minimally invasive transforaminal lumbar interbody fusion result in superior outcomes? World Neurosurg 2015;83:860-6. 